\title{
El amor y la consulta. Relaciones amorosas y problemas médicos
}

\author{
R. ManRique Solana \\ Psiquiatra. Práctica Privada. Santander
}

\section{RESUMEN}

El artículo trata de la articulación entre la psi cología y los problemas de las relaciones amorosas y la sintomatología física.

La evolución social está cambiando las relacio nes familiares y las relaciones amorosas. Al tiempo los problemas, conflictos o trastornos de personali dad están aumentando. Ambas cosas alteran la ca pacidad de establecer relaciones amorosas sólidas y estables. Mucho del sufrimiento físico que llega a la consulta del médico tiene que ver con problemas de amor y desamor. El médico debe de conocer es tos problemas para ayudar a sus pacientes a inter pretar su patología y buscar soluciones.

Palabras clave: Familiar. Relaciones interper sonales. Amor. Trastornos de personalidad.

Amar es sufrir No amar es enfermar

\section{S. Freud}

\section{LA FRAGMENTACIÓN DE LAS RELACIONES AMOROSAS EN EL TRABAJO DEL MÉDICO DE FAMILIA}

Una de las habilidades terapéuticas del médico de familia ha de ser disponer de lo que, en términos
The love and the consult. Loving relations and me dical problems

\begin{abstract}
This paper deals with the connection between loving relationships and physical symptoms. Social evolution is changing family and loving relationships. At the same time interpersonal conflicts and personality disturbances are increasing. All these things create problems in the ability to establish clear and stable loving relationships.

The general practitioner works with a lot of pa tients who suffer psychologically and physically due to these emotional problems. The physician needs to know the patient's situation in order to help them and to interpret their pathology and look for solutions.
\end{abstract}

Key words: Family. Interpersonal relations. Love. Personal disorders. tradicionales se ha llamado ontología, esto es, una teoría acerca de lo que es el ser humano. Aun sin ser muy consciente de ella es seguro que al médico le va a servir de base a su trabajo e incluso, a veces, le va a ser directamente aplicable a su estrategia terapéutica. La dinámica familiar en general y la de pareja que en este artículo vamos a contemplar, pertenece a esa dimensión.

Vivimos, cada vez más, en un mundo en el que las formas tradicionales de vida se han fragmentado, y se han producido múltiples estilos de relaciones humanas. Las relaciones sociales se basan mu- 
cho más en lo individual, y es previsible que las relaciones, familiares, amorosas y sexuales sean también multiformes y se centren en lo personal y no en lo colectivo. De hecho, ya la familia tradicional, compuesta de padre, madre y dos hijos, está en minoría en algunos países respecto a las familias con hijos adoptivos, segundos matrimonios, relaciones entre solteros que viven de forma independiente uno del otro, personas que viven juntas sin casarse, parejas de homosexuales,... Y si la familia cambia el trabajo del médico de familia también lo hará. Las estadísticas nos dan datos muy significativos. Tomando el periodo 1970-1995 se observa que la tasa de divorcios se ha duplicado en muchos de los países occidentales. Esta tendencia al aumento de los divorcios se advierte con mayor o menor importancia en todo el mundo. Y eso sin incluir las separaciones de facto o de las parejas no casadas. El retraso en la edad de matrimonio también es algo universal. La proporción de personas no casadas ha aumentado. Asimismo lo ha hecho el número de hijos que se tienen fuera del matrimonio. Todo ello nos indica que hay, entre otros factores, un malestar en la forma tradicional en que se han organizado las relaciones amorosas. Pero también nos indica que hay un intento en marcha de construir nuevos modelos de relación humana. Y todo esto no pertenece al campo de la teoría sociológica sino que está en la esencia misma de la experiencia de vida de las personas que acuden a la consulta. Y si es así estas realidades forman parte del bagaje necesario para el médico de familia.

Los médicos trabajan con pacientes que viven en alguna situación respecto a las relaciones amorosas. Sufren o disfrutan por ellas; por su presencia o por su carencia. El amor, las relaciones amorosas y las familiares, forman parte del nicho en el que nuestro malestar -o bienestar- físico y mental se genera. Y en nuestro mundo occidental ese sufrimiento, ese malestar, se suele expresar en forma de enfermedad o síntoma físico. La enfermedad es una forma socialmente aceptable de sufrir y para la que se dispone de un dispositivo de acogida: los servicios médicos en cualquiera de sus niveles pero especialmente en el nivel primario, en el de la medicina llamada de familia.

\section{ALGUNOS CONCEPTOS SOBRE LA RELACIÓN AMOROSA DE PAREJA}

No deja de ser sorprendente que -y a mi juicio es una grave carencia- el sistema de medicina familiar tienda a dejar de lado la comprensión e intervención en ese sistema humano básico -la relación de pareja- a la hora de entender la enfermedad de una persona. En consecuencia se obvia uno de los más importantes sistemas generadores de pro- blemas médicos. Quizá sea debido a la dificultad de operar con esa entidad tan ambigua y cambiante como es la pareja y el amor, con todo el conjunto de realidades sentimentales y materiales que en ella se generan.

Me gustaría llamar la atención en este artículo precisamente acerca del problema de las relaciones amorosas y su incidencia en los problemas médicos. Creo que la comprensión de estas dinámicas facilitará el abordaje médico de muchos problemas que se observan en el consultorio.

Y para ello es necesario referirnos a algunas características de la pareja humana. Una primera distinción, obvia pero necesaria, es que una cosa es el matrimonio y otra el amor. El matrimonio es una institución social destinada a organizar alrededor de ella una familia y un sistema de reproducción de la realidad social. Adopta la forma de contrato. El amor, sin embargo, es una relación, un intercambio, es algo basado en sentimientos que siempre son frágiles.

El caso es que lo institucional y lo relacional no se llevan bien y con frecuencia el matrimonio ahoga el amor como sentimiento y esto va a tener mucho impacto el el equilibro psicofísico de las personas.

Quizá lo primero que podamos saber acerca de nosotros mismos sea que tenemos algún sentimiento: aburrimiento, tristeza, ira, ansiedad, alegría,... Siempre estamos en algún estado sentimental. Tener emociones y vivir en estados sentimentales hace posible la vida. No existen porque permitan conocimiento o placer, sino por su utilidad para la supervivencia. Los sentimientos son un fenómeno mental muy particular. Pueden ser comprendidos como disposiciones para la acción y, al tiempo, como sistemas de evaluación del estado actual de un ser. Por ello al interrogar a un paciente sobre sus sentimientos nos estamos haciendo una idea clara de cómo ve el mundo desde su punto de vista en ese momento concreto. Y de esa visión de sí y de sus circunstancias se derivan muchas de las realidades que afectan a una persona, entre ellas su estado físico. De entre todos los sentimientos el amor es el más importante. Correlaciona directamente con muchas situaciones humanas. Una de ellas con la salud y la enfermedad. Volveremos luego a ello. Antes continuemos nuestra reflexión sobre el amor. De la pegajosidad biológica, natural podríamos decir, de los mamíferos surgen dos fenómenos muy importantes: el amor y la ternura. La ternura implica hacerse cargo de las disposiciones sentimentales del otro, acogerlas y protegerlas. Esta atención hacia el otro combina la suavidad física y emocional con una neutralización de los afectos negativos, que están siempre presentes en cualquier relación. Por eso la ternura es, a veces, una simbolización de los ataques físicos: un beso en la nuca, un suave 
mordisco, "comer" a besos,... La persona tierna está en control de su mundo cognitivo y emocional y lo ofrece al otro. Por eso es tan apreciada, por lo genuina. Muchas cosas se pueden fingir; la ternura, apenas, ya que atraviesa todas las capacidades de la persona. Sin amor, sin ternura, se enferma y se mal vive.

Ambos fenómenos son muy apreciados entre los humanos. Sin ellos la vida es casi imposible o es un horror. Muchas veces a los médicos les pasa desapercibido que su paciente, que tiene este o aquel síntoma o enfermedad, está viviendo en la carencia de ambos. Sin amor y sin ternura la vida es difícil y la enfermedad una amenaza pero también, a veces, un consuelo. Su falta o su alteración nos predispone a acciones muchas veces autodestructivas: drogas, alcohol, accidentes, alteraciones funcionales, etc. También nos hace evaluar la vida como un destino deprimente o amenazador. Con todo ello van las personas a la consulta del médico.

El amor, centrándome en el amor adulto, consiste en una relación comprometida y erótica que establecemos con otra persona. Se concibe como perdurable en su esencia, y, al tiempo, se muestra ella misma en su andadura como frágil y difícil de mantener.

Existen tres tipos de problemas en lo que se refiere a la relación amorosa:

-Derivados del ciclo vital:

A lo largo de una relación amorosa se recorre un ciclo con etapas más o menos definidas y por las que siempre se pasa con mayor o menor acierto. $\mathrm{Y}$ en esas etapa se dan unos puntos críticos de cuya resolución depende el bienestar personal y el desarrollo armónico de la relación. Son muy conocidos y quizá por ello se pasa por alto su importancia. Se trata de los primeros tiempos de vida en común, de la llegada del primer hijo, de la partida de los hijos, de la entrada de los cónyuges en la década de los cuarenta y la jubilación, por señalar los más importantes. No son situaciones anormales, no dependen de cómo sea cada persona sino que siempre se pasa por esas etapas. Pero que sean normales y necesarias no significa que no sean difíciles y que sus dificultades se expresen delante de la mesa del médico como problemas de salud y no como problemas de relación respecto al compromiso amoroso o al erótico.

-Derivados de los cambios sociales:

Lo que se esperaba de los hombres y de las mujeres, lo que se esperaba de la vida en común ha cambiado bastante. Especialmente los hombres están desorientados. Ser "machos" ya no sirve, pero, entonces, ¿qué sirve? ¿qué diferencia a un hombre de una mujer ?... La llamada "cuestión de género" se ha convertido en uno de los temas más importantes en el campo del análisis social pero también desde el campo de la salud. Muchos problemas de orden sexual y de orden médico tienen que ver con la angustia de los hombres que se sienten perdidos e incomprendidos en esta nueva situación.

Pero no sólo está la crisis del modelo patriarcal sobre el que se han asentado la familia y el amor. Otros factores muy importantes están creando problemas en el desarrollo del amor: el desempleo, la presencia de los hijos en casa hasta edades muy avanzadas, la precariedad en el trabajo, la carestía de la vida... todo ello tiene un impacto muy grande en la definición que cada uno se da de sí mismo, en su capacidad de establecer relaciones y en consecuencia cambia el modo de amar y surgen las dudas, las angustias, los problemas,... la enfermedad.

-Derivados de la estructura personal de los cónyuges:

Y junto a ello están las dificultades personales de cada uno, lo que podríamos llamar problemas de personalidad. No podemos detenernos en su dinámica específica pero baste señalar que los déficits, conflictos o problemas estructurales de la personalidad van a crear sesgos importantes en el modo de relación amorosa que se vive.

\section{LA RELACIONES AMOROSAS QUE EL MÉDICO OBSERVA}

Y toda esa mezcla llega al médico envuelta en un problema de salud, en una demanda de curación. Con frecuencia será evidente al médico que la relación matrimonial está jugando un papel en la enfermedad o problema de su paciente. De una manera casi intuitiva podemos encontrar cuatro grandes modos de relación amorosa:

1. Relación asimétrica.

Se trata de relaciones en las que cada miembro de la pareja realiza un papel o una conducta que complementa aspectos que encajan en las del otro. Las funciones están claramente divididas. Uno es quien sabe; el otro, el que aprende. Uno es organizador; el otro, organizado. Uno desempeña el papel social de hombre; el otro, el de mujer. Uno es activo; el otro, pasivo... el objetivo de la relación es el mantenimiento de la relación misma.

2. Relación coordinada.

Consiste en que cada miembro de la pareja posee una identidad diferenciada que comparte y, en ocasiones, se adapta a la del otro. Las funciones de cada uno son intercambiables, están solapadas. El objetivo es el desarrollo, el despliegue de cada personalidad, pero dentro de los límites que permitan el mantenimiento de su relación. Se trata de una relación abierta al exterior.

3. Relación formal.

En este tercer modo de relación, cada miembro de la pareja realiza un guión que ya está establecido por el otro, la familia o la sociedad. El hombre 
se comporta, por ejemplo, como un caballero y la mujer como una princesa. La forma y la estética de la relación son más importantes que la relación o las personas mismas.

4. Relaciones objetales.

Son relaciones en las que uno de los miembros de la pareja o los dos son, meramente, un objeto, un instrumento, para el uso del otro o para el cumplimiento de los fines del otro. El ejemplo más clásico es el matrimonio de conveniencia. La personas son, en esta relación, un medio económico.

Todas estas formas de relación tienen ventajas e inconvenientes. Todas pueden ser estables o frágiles. Pero no todas son igualmente deseables desde el punto de vista ético. Las relaciones objetales, por ejemplo, pueden acabar fácilmente en violencia. Las complementarias o asimétricas acaban en una rutina mortecina. Las coordinadas pueden acabar en el desamor y las formales son empobrecedoras y rígidas. Respecto a las ventajas, podemos decir, a modo de rápido ejemplo, que la asimétrica permite la compensación de carencias; la coordinada, el despliegue de la originalidad; la formal, la seguridad; y la relación objetal puede permitir escapar de alguna situación familiar o personal horrible.

\section{INTERVENCIÓN MÉDICA EN UN MARCO DE CONFLICTO AMOROSO}

Desde luego estos modelos tienen que ver con las formas de enfermar pero hay que decir que la compresión de la dinámica matrimonial es muy difícil. Junto a lo observable, a lo aparente, hay un mundo no conocido de sentimientos, expectativas, fracasos que es importante conocer y que hace que haya que ir con sumo cuidado cuando se trata con un problema matrimonial. He señalado ya que la relación de pareja se base en dos pilares. El compromiso y el erotismo. Casi todas las quejas o dificultades que se escuchan tienen que ver con estas áreas. Divergencias en lo erótico (cansancio, rutina, desacoplamiento sexual, falta de deseo) o en el compromiso (diferentes responsabilidades, dedicación a otras tareas, "agendas secretas"). En mi opinión el médico ha de desarrollar diversas habilidades.

-Mantener una actitud de apertura hacia la detección de la expresión de los problemas de relación matrimonial en sus pacientes.

- Conocimiento de las tareas del ciclo vital y los puntos de transición -como los que he mencionado más arriba- de la familia que correlacionan directamente con el enfermar.

-Establecer correlaciones entre los problemas de personalidad y los modelos amorosos que he esbozado.

- La anamnesis médica debe de preguntar por el tipo de relaciones amorosas. Especialmente el compromiso y el erotismo deben de ser investigados de la manera más apropiada dentro de los límites y características de una relación médica como la que se da en campo de la medicina de familia. Hay siempre una compleja realidad emocional detrás de todo enfermar pero sobre todo en aquellas enfermedades de tipo crónico o funcional.

- Proponer, según venga el caso, a sus pacientes la conexión que puede haber entre sus realidades emocionales y amorosas y sus síntomas físicos.

- Intervenir en aquellas realidades de la situación emocional del paciente que sean susceptibles de cambio desde el trabajo médico.

-Finalmente y en caso de necesidad, el médico debe de remitir al paciente a un especialista en terapia de pareja y ser capaz de hacer esa recomendación en un ambiente de colaboración y respeto.

Todo esto no significa que el médico sea un trabajador social o un psiquiatra. Significa solamente que la enfermedad es una realidad biológica, psicológica y social. El médico debe de poder intervenir, o hacer que se intervenga, en todo aquello que esté en su mano y que esté conectado con la curación o alivio de los problemas de sus pacientes. Tan médico y tan importante es dar un antibiótico como asegurarse de la calidad del agua, la alimentación de sus pacientes o su vida emocional. Todo ello hace la labor médica más compleja pero también más fascinante.

Una viñeta pueden ilustrar estos temas. Hace poco y de una manera informal tuve la ocasión de observar la importancia de esta comprensión. Hablaba, en un descanso de una reunión, con una colega médica de familia de una persona que era su paciente y a la que yo conocía. Se trataba de una mujer que había sido diagnosticada de fibromialgia. Pasemos por alto el hecho de que sea este un diagnóstico un tanto incierto. La paciente había sido tratada de sus múltiples síntomas sin ningún éxito. Era un mujer inteligente y batalladora y mi colega estaba algo desesperada y ya sin recursos. Como yo conocía a su paciente le comente algo de la situación amorosa por la que atravesaba esta mujer. Nada dijimos que fuera llamativo o espectacular sino que fueron unos pocos comentarios sobre su trayectoria amorosa los que cambiaron la comprensión de mi colega. Los datos que tenía acerca de ella se conectaron repentinamente. Era como si todo adquiriera significado. Quedó en llamarme cuando viera a esta persona en su consulta. Así hizo. Tres semanas después me llamó y me comentó cómo había enfocado las consultas con ella. Había abordado directamente su vida amorosa que en los últimos tiempos había sido un desastre dentro del descalabro general sentimental en el siempre había vivido. Se daba asimismo por este tema un fuerte enfrentamiento con su padre que, al tiempo, era su jefe en la empresa familiar. En los últimos meses se 
habían sucedido problemas que con sólo mencionarlos la paciente se alteraba y parecía evidente que llevaba un tiempo repitiendo un tipo de relación amorosa con los hombres muy negativa. El médico descubrió entonces una situación de conflicto que le hacía sufrir mucho y que era necesario abordar. La fibromialgia podía ser entendida como una especie de tregua en esa batalla con los hombres que el médico señaló. El cansancio que lleva aparejado había supuesto, paradójicamente, un descanso al que no iba a renunciar sin más. Hablar de ello siquiera brevemente fue muy útil e inauguró un nuevo estilo de relación médico-paciente. No es que la fibromialgia fuera a desaparecer por arte de magia pero la paciente asumió que era posible vivir con esas molestias y abordar algo de su conflictiva vida sentimental en la seguridad que estaban conectadas y que una y la otra habrían de mejorar simultáneamente.

El objetivo de amar y sentirse amado, al que tantos esfuerzos se dedican, puede salir mal. Y enton- ces nos instalamos en unos estados sentimentales dolorosos: fracaso, decepción, resignación, reproches,... que son difíciles de sobrellevar. Freud decía que amar es sufrir, pero no amar es enfermar. No ser, o no sentirse, amado, puede hundir a una persona en la depresión, en la amargura y en la enfermedad. Pero también, como toda experiencia, puede servir de base para una reflexión que permita a una persona cambiar la propia trayectoria vital y en consecuencia mejorar su salud.

\section{CORRESPONDENCIA:}

Rafael Manrique Solana

Gómez Oreña, 15, 2

39003 Santander

e-mail: rmanrique@teleline.es

\section{Bibliografía recomendada}

1. Bolwby J. Una base segura. Barcelona: Paidós, 1993.

2. Carter B, Mcgoldrick M. The changing family life cycle. Boston: Allyn and Bacon, 1989.

3. Foucault M. Historia de la sexualidad, 3 Vols I, Madrid: siglo XXI, 1987.

4. Freud S. Algunos mecanismos neuróticos en los celos. La paronoia y la homosexualidad. Obras completas, tomo XVIII. Buenos Aires: Amorrortu, 1976 (1922).

5. Gurmendez C.Sentimientos básicos de la vida humana. Madrid: Libertarias-Prodhufi, 1994.

6. Manrique R. Sexo, erotismo y amor. Madrid: Libertarias, 1996.
7. Montreynaud F. Amar. Barcelona: Evergreen, 1998.

8. Moultrup DJ. Husbands. Wives and lovers. Nueva York: Guilford, 1990.

9. Paz O. La llama doble. Erotismo y amor. Barcelona: Seix Barral, 1993.

10. Reich W. La revolución sexual. Buenos Aires: Paidós, 1980.

11. Rougemont D. Amor y occidente. Barcelona: Kairós, 1986.

12. Rubern R. El eros electrónico. Barcelona: Taurus, 1999.

13. Skinner R, Cleese J. Families and how survive them. Londres: Methuen, 1983.

14. Vidal G. Sexually speaking. San Francisco: Cleis Press, 1999. 